\title{
Research Paper \\ Effect of Hip Abductor Muscle Fatigue on Static and Dynamic Balance in Elderly Women
}

\author{
*Farideh Babakhani ${ }^{1}$, Kobra Oladghobadi², Farajollah Fatahi ${ }^{3}$
}

1. Department of Pathology and Corrective Exercises, Faculty of Physical Education and Sports Sciences, University of Allameh Tabataba'i, Tehran, Iran.

2. Department of Pathology and Corrective Exercises, Faculty of Physical Education and Sports Sciences, University of Razi, Kermanshah, Iran.

3. Department of Pathology and Corrective Exercises, Faculty of Physical Education, University of Isfahan, Isfahan, Iran.

Received: 8 Apr. 2016

Accepted: 12 Jun. 2016

\section{A B STRACT}

Objectives Postural control is the act of maintaining and restoring a better state of balance and proper biomechanics structure in limbs and different parts of the body. Such proper structure exists in two forms namely static and dynamic. Fatigue is one of the factors that affect the postural control and body stability. The purpose of this study was to examine the effect of hip abductor muscle fatigue on the static and dynamic balance in elderly women.

Methods \& Materials The participants of this study included 25 elderly and healthy women without any disability $(60.24 \pm 8.5$ years old, weight: $60.58 \pm 9.54 \mathrm{~kg}$, height: $153.42 \pm 7.65 \mathrm{~cm})$. In the first session, the participants underwent static and dynamic balance tests in the normal condition (without fatigue). In the second session, at first, they participated in the hip abductor muscle fatigue protocol followed by static and dynamic balance tests. In addition, t-test was used to compare the results before and after the fatigue.

Results The results indicated that the hip abductor muscle fatigue protocol led to a significant reduction in test scores of balance tests, which included static and dynamic balance. Also, a significant difference was observed between static and dynamic balance before and after fatigue. Overall, the measure of the effect of fatigue protocol was notable in both the tests $(P<0.05)$.

Conclusion Reduction in the scores of static and dynamic balance tests showed that the hip abductor muscle fatigue caused an increase in the amount of damage and length of disability in elderly women.

\section{* Corresponding Author:}

Address: Department of Pathology and Corrective Exercises, Faculty of Physical Education and Sports Sciences, University of Allameh Tabataba'i, Dehkade Olampic, Shahid Hemmat Hwy., Tehran, Iran.

Tel: +98 (21) 44118630

E-mail: farideh_babakhani@yahoo.com 


\title{
تأثير خستكى عضلات دوركننده ران بر تعادل ايستا و بوياى زنان سالمند
}

\author{
"فريده باباخانى'، كبرى اولادقبادى '، فرجالله فتاحى" \\ ا- كروه آسيبشناسى ورزشى و حركات اصلاحى، انشكده تربيت بدنى و علوم ورزشى، دانشكاه علامه طباطبايى، تهران، ايران.

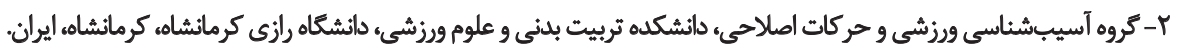

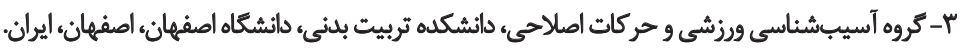

\begin{abstract}
حكSد

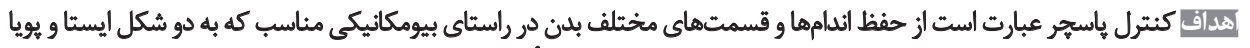

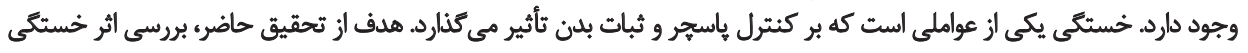

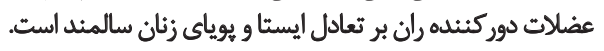

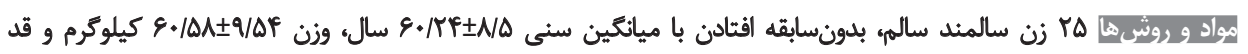
هIF

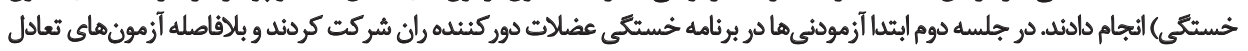

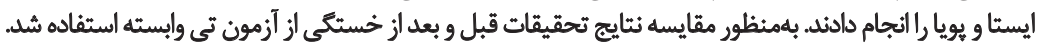

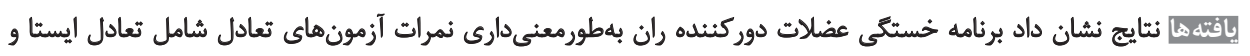

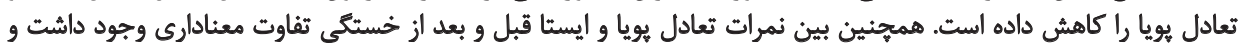

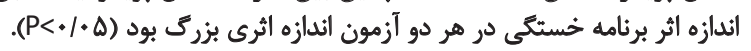

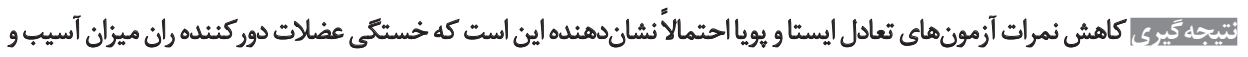

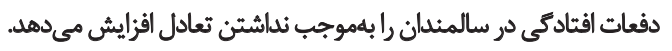

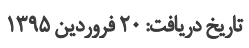

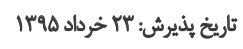

كليدوارٌها:

عضلات دوركنينده رانه

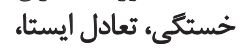
تعادل يويا، زنان سالميند

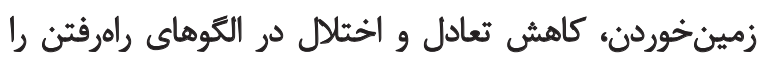

مقدمه

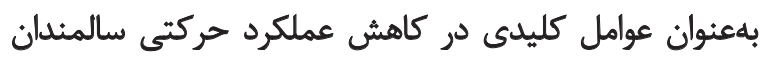

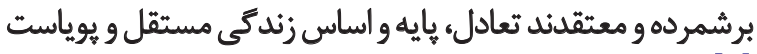

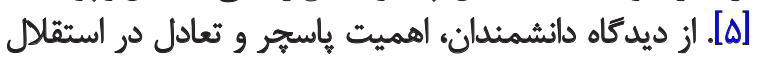

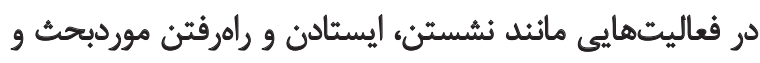
غيرقابل|نكار است.

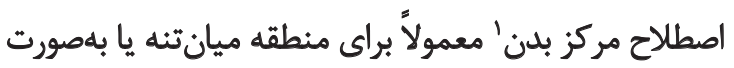

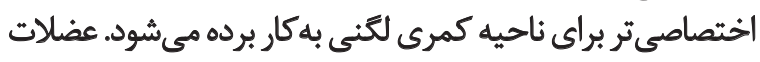

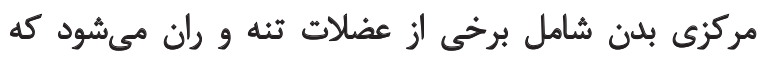

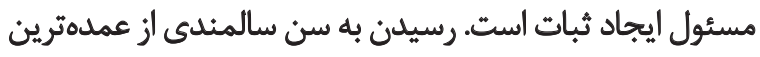

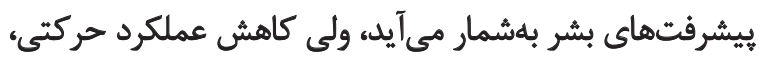

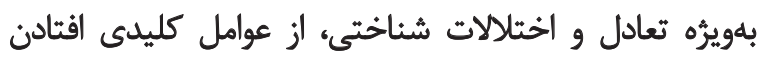

1. Core

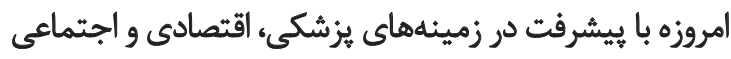

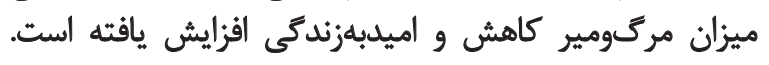

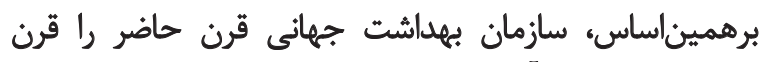

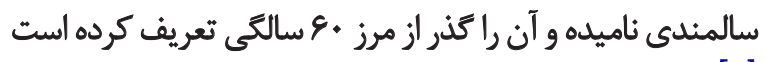

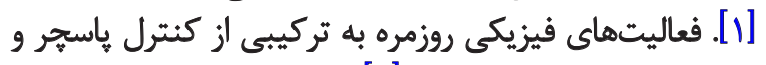

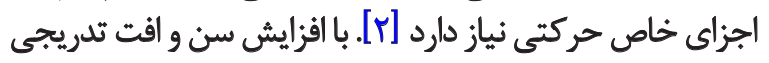

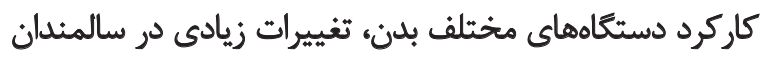

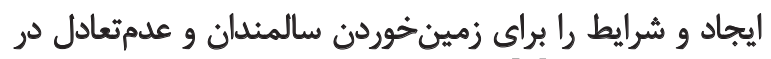

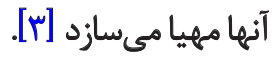

افتادن يكى از شايعترين و جدى مترين مشكلات مات دوران

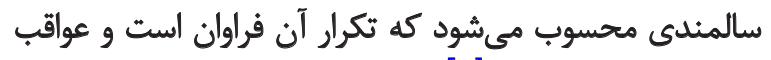

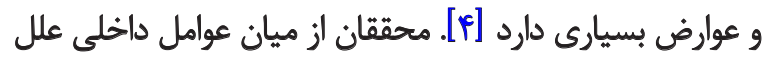

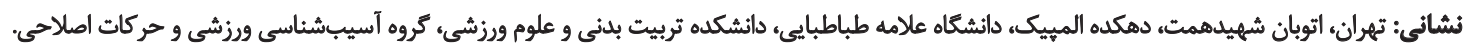

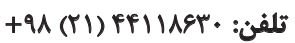
يست الكترونيكي: farideh_Babakhani@yahoo.com 
يك بروتكل در عضلات دوركنيده ايجاد شد)؛ بنابراين، عنوان

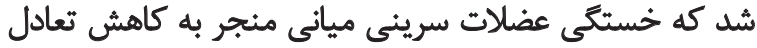

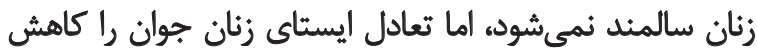

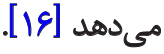

باوجوداين، كريبل ارتباط معنادارى بين خستكى عضلات

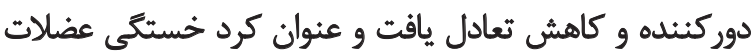

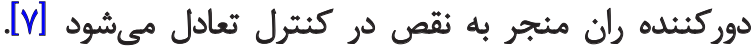

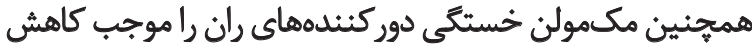

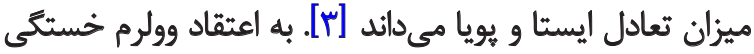

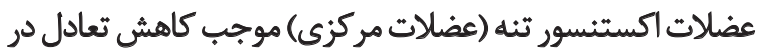

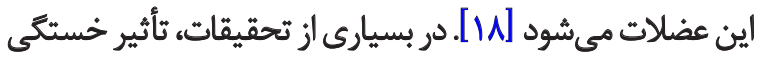

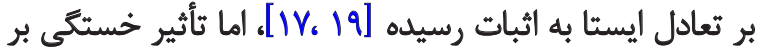

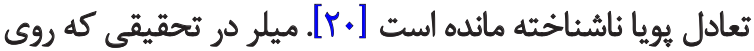

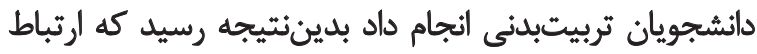

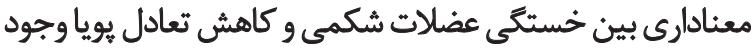

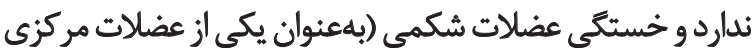

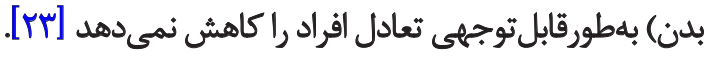
ستين خستكى رادر اندام تحتانيى و تنه ايجاد كرد و به سنجش

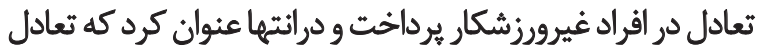

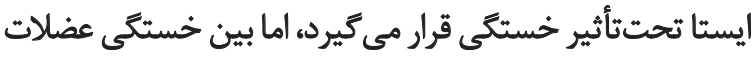

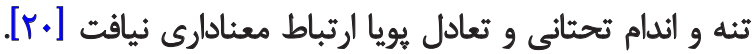

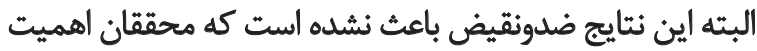

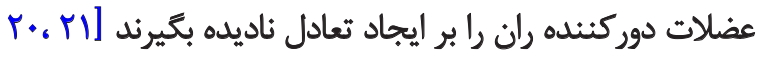

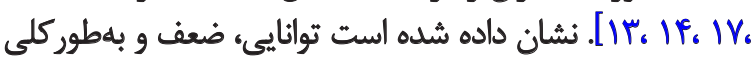

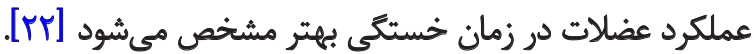

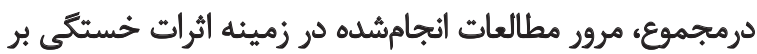

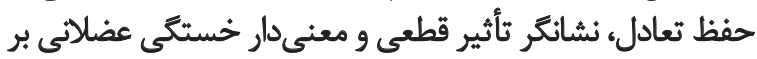

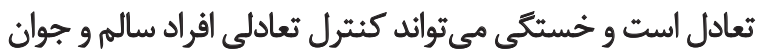

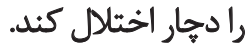

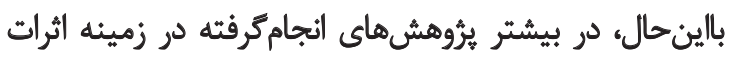

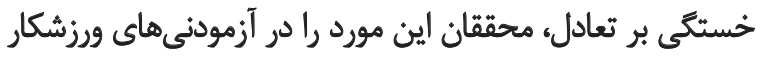

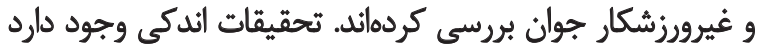

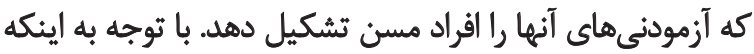

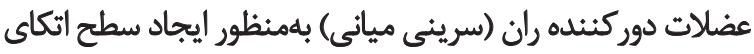

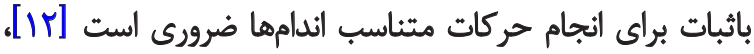

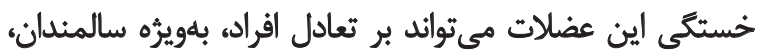

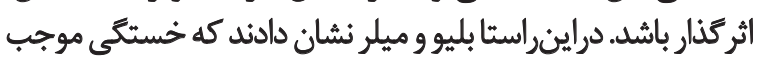

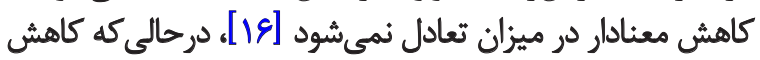

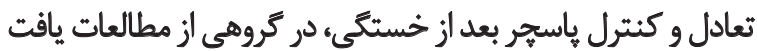

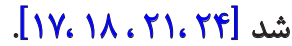

ثبات مركزى و عضلات دوركننده ران مي تواند نقش تأثير كنارى

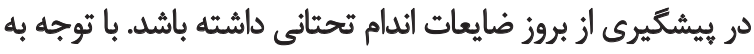

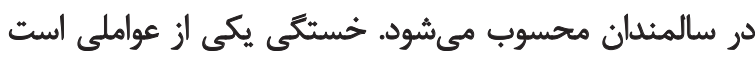

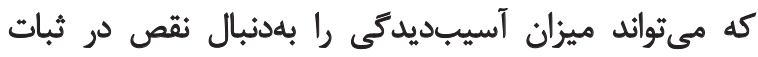

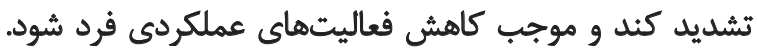

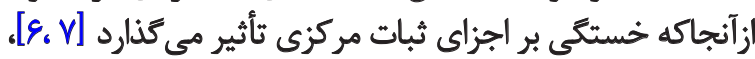

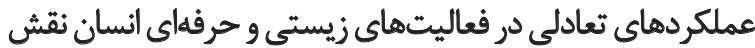

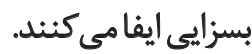

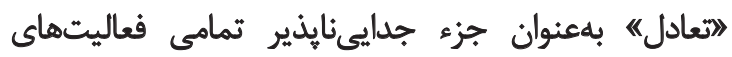

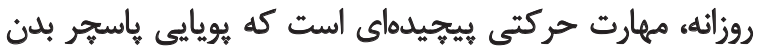

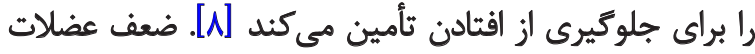

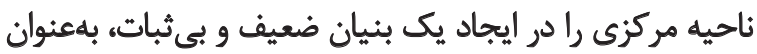

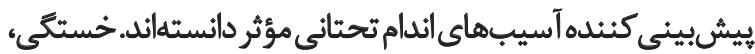

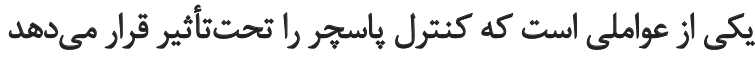

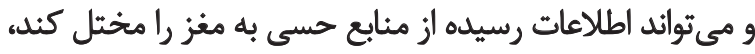

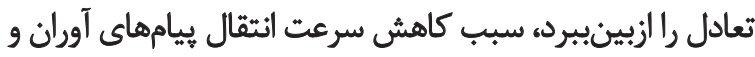

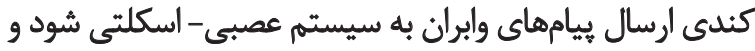
بر توانايى حركات مؤثر جبرانى اثر منفى مي كذارد [9]

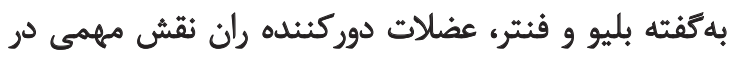

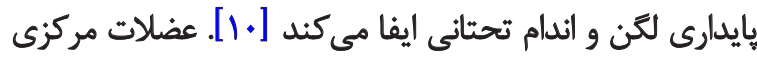

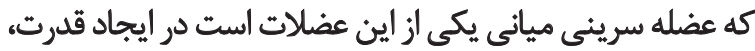

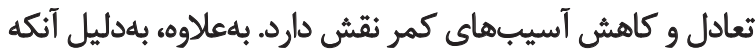

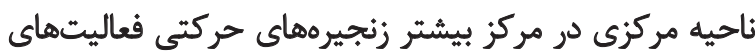

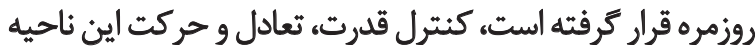

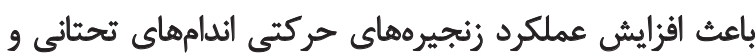

فوقانى و عملكرد مى فشود [ili]

عضله سرينى ميانى، اصلىترين دوركنيده ران و مهارمترين

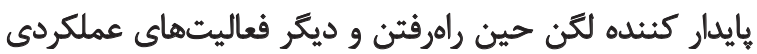

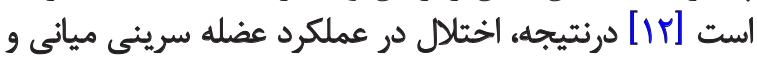

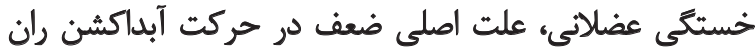

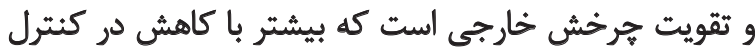

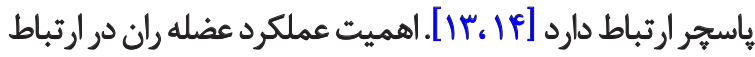

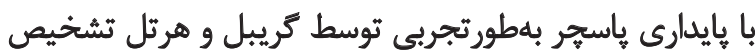

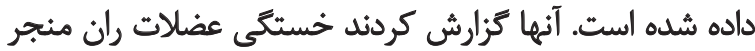

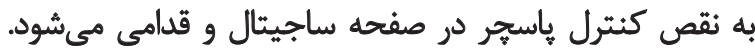

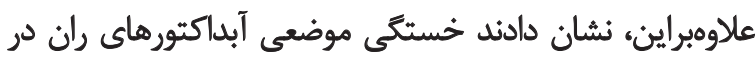

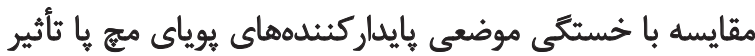

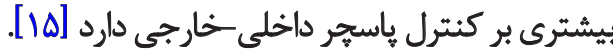

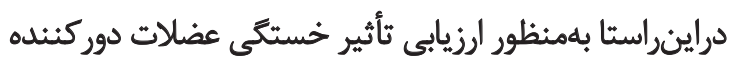

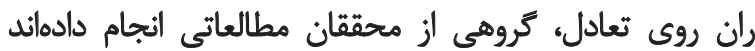

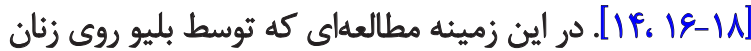

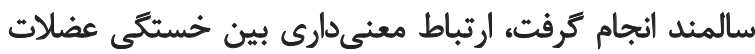

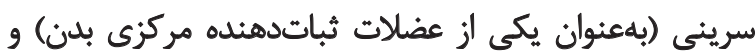

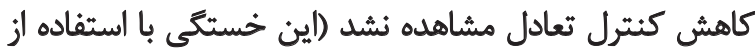


نجوه اجرائ أزمونها

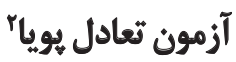

اين آزمون براى سنجش تعادل يويا در عضلات آبداكتور ران

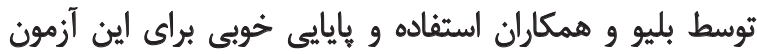

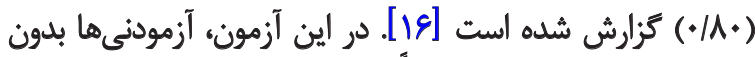

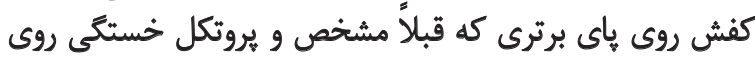

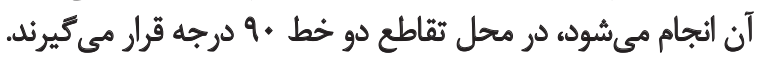

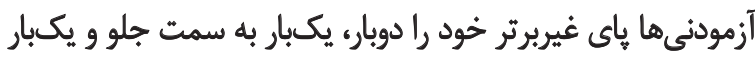

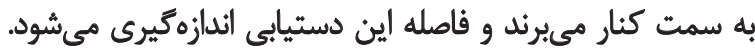

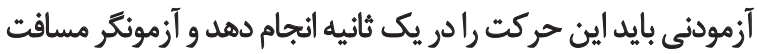

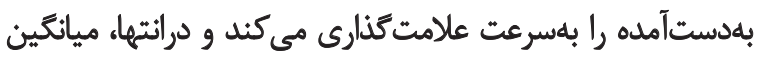

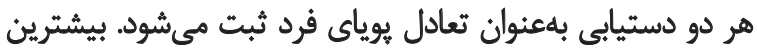

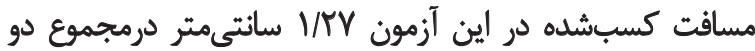

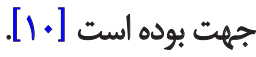

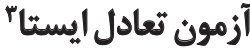

اين آزمون براى سنجش تعادل ايستا در عضلات دوركنينده

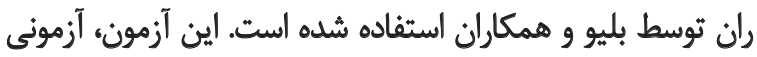

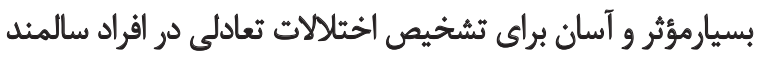

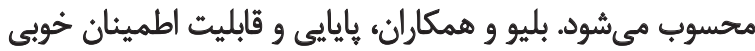

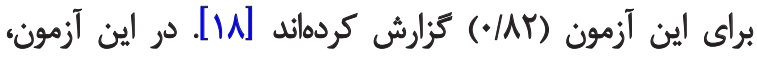

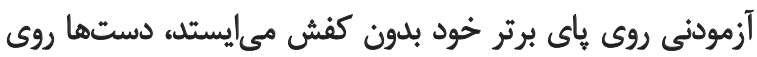

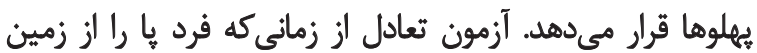

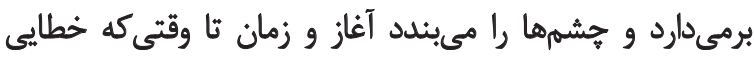

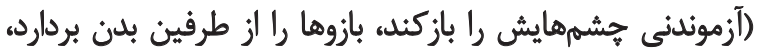

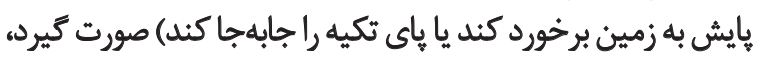

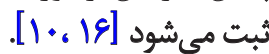

\section{يروتكل خستكى عضلات دوركنيده ران}

در اين مطالعه از يروتكل خستخى ياتريك و همكاران براى

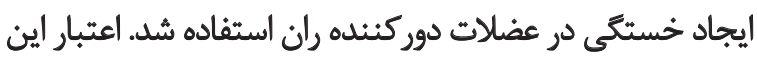

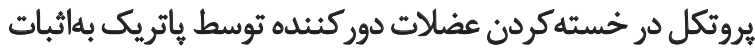

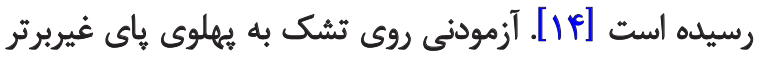

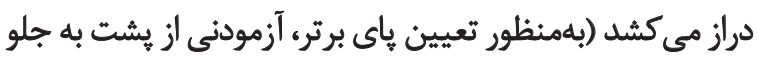

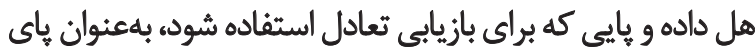

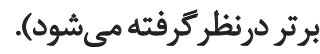

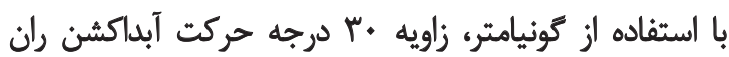

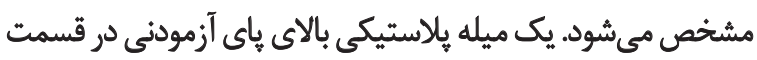

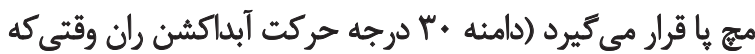

2. Lower-extremity reach test

3. Single-limb stance time test
اينكه ناكنون جنين تحقيقى در جامعه ايرانى روى زنان سالمند

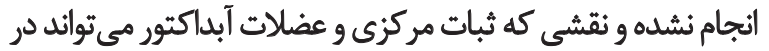

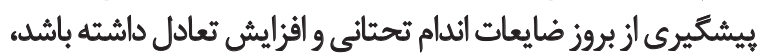

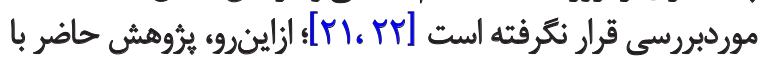

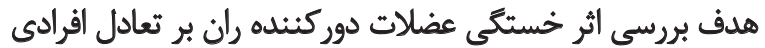

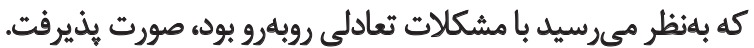

\section{روش مطالعه}

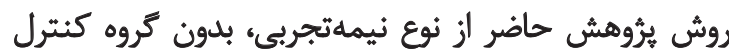

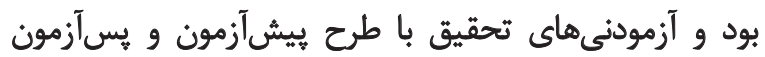

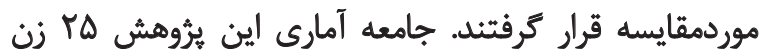

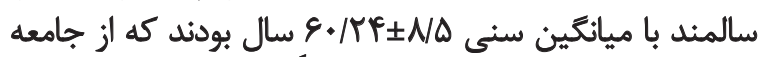

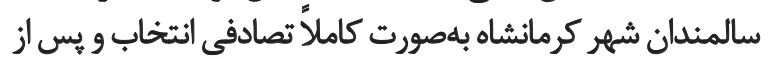

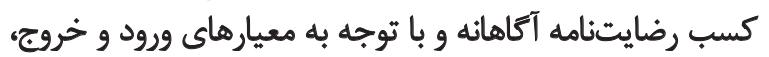

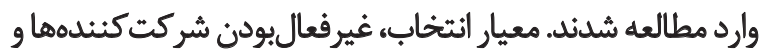
فقط شركت در فعاليتهاى روزانه بود.

معيارهاي ورود به تحقيق جنسيت مؤنث و سن بالاتر از •وسال

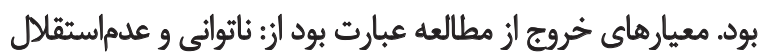

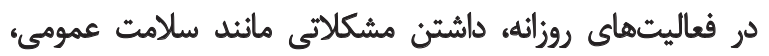

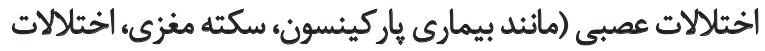

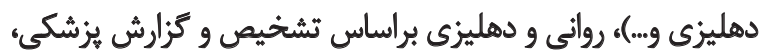

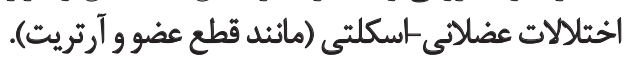

تعداد جلسات آزمون Y جلسه (فاصله ميان جلسات حداقل

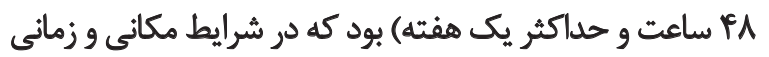

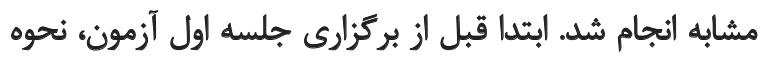

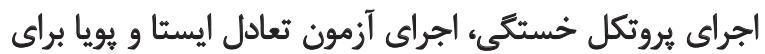

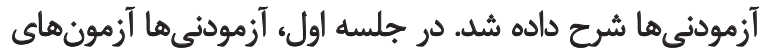

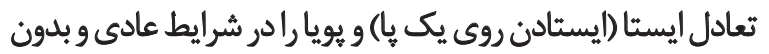

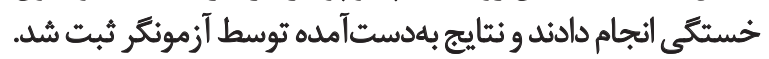

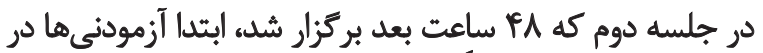

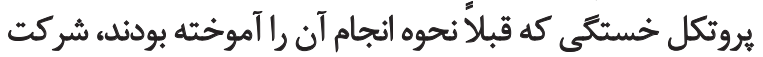

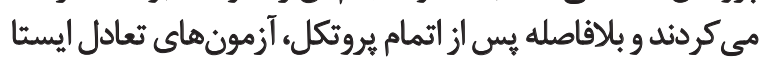
و يويا را انجام مي دادند.

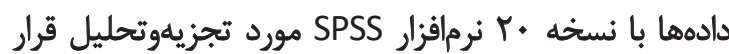

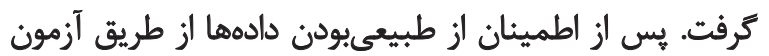

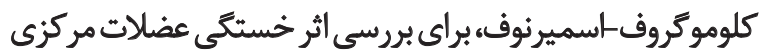

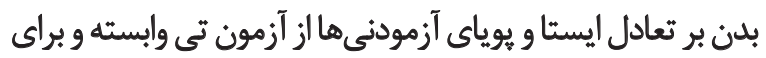

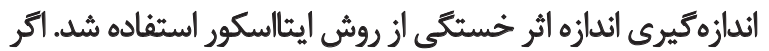

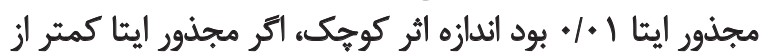

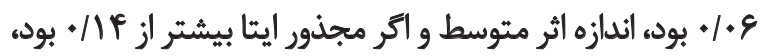

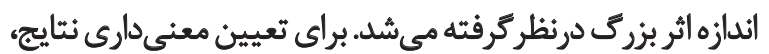

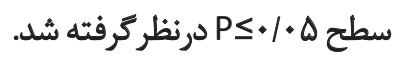


سن، قد، وزن در يرؤهش، در جدول شماره ا نُشان داده شده است. جدول شماره r، نتايج حاصل از آزمون تى وابسته را نشان

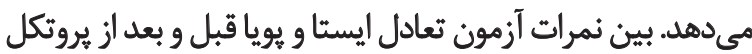

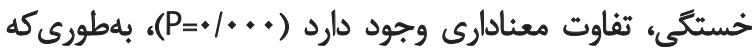

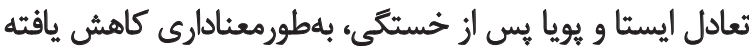

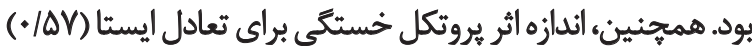

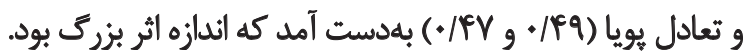

بحث

هدف از يثروشش حاضر، بررسى ثأثير خستكى عضلات

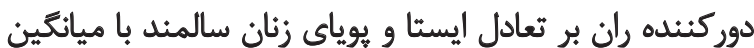

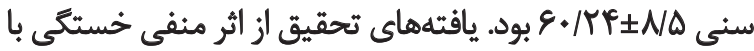

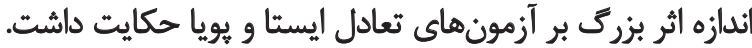

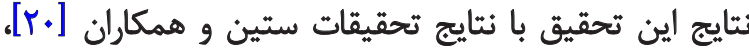

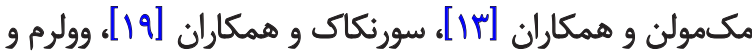

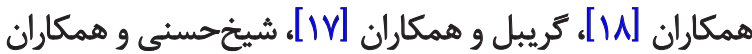

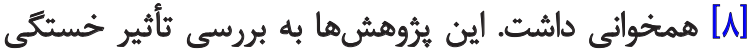

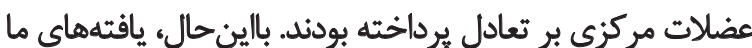

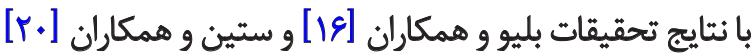

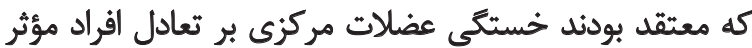

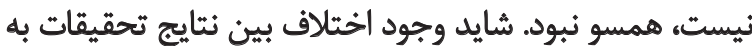

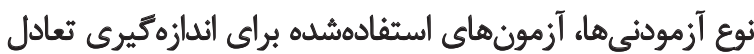

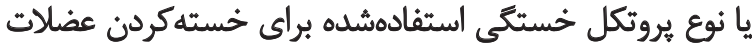

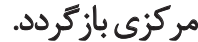

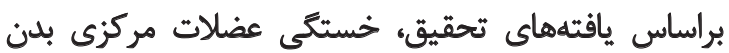

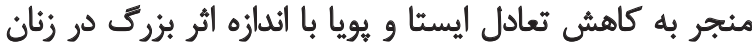

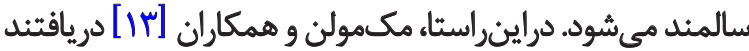

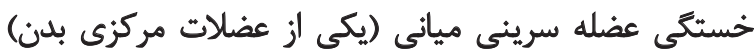

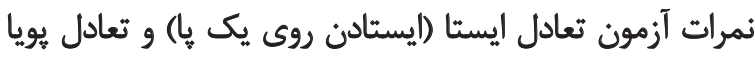

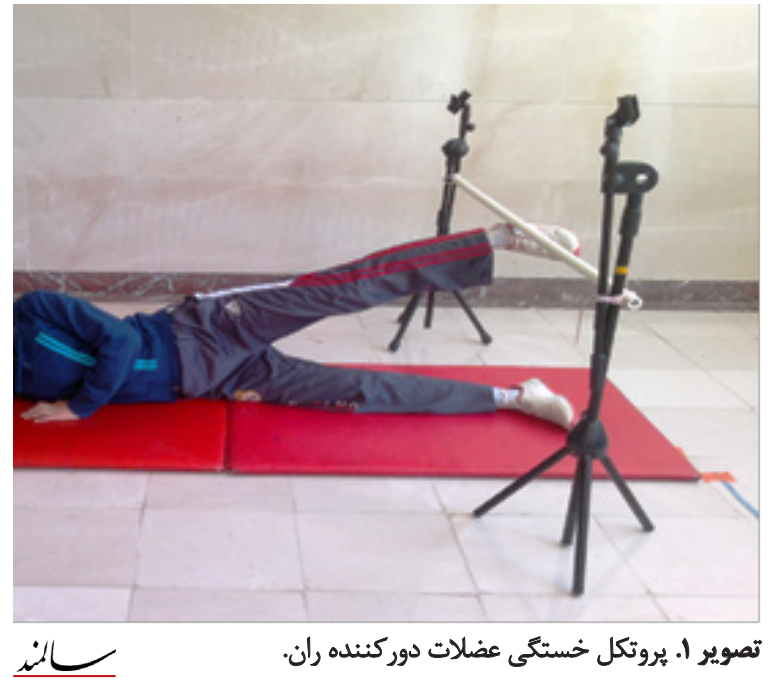

زانوى آزمودنى در حالت اكستنشن كامل است مشخص مي مشود و

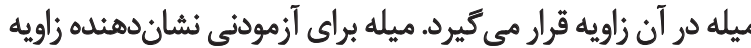

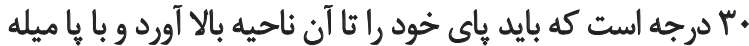

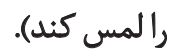

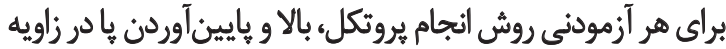

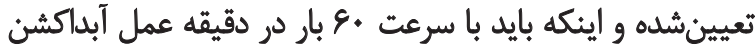

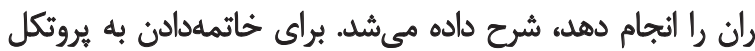

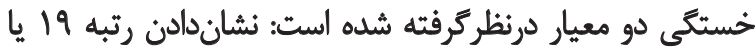

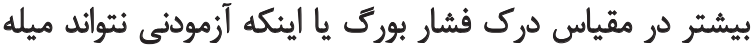

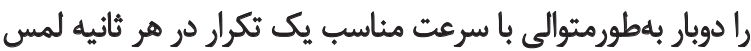

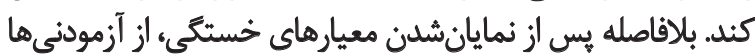

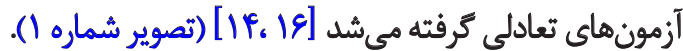

يافتها

اطلاعات توصيفى مربوط به مشخصات شركتكنئدكان شامل

جدول ا. مشخصات عمومى آزمونئىها.

\begin{tabular}{|c|c|c|}
\hline سن (سال) & وزن (كيلوكَرم) & قد (سانتىمتر) \\
\hline$\varepsilon \cdot / \pi f \pm N \Delta$ & $9 \cdot / \Delta A \pm V / \Delta F$ & $\mid Q T / F T \pm V / \& \Delta$ \\
\hline
\end{tabular}

ك

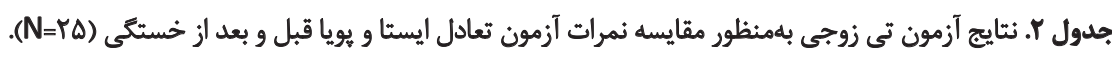

\begin{tabular}{|c|c|c|c|c|c|c|}
\hline اندازه اثر & P-value & Tاري t t t & تفاوت ميانغين ها & يس آزمون & ييشي آزمون & آزمونهاي تعادل \\
\hline .prer & $.1 . . *$ & ples & $r / r \pm r g / \Delta r$ & $V / q \pm \cdot N \Delta r$ & $V / A \pm R T / A S$ & تعادل يويا قدام \\
\hline$+/ 19$ & $.1 . . *$ & p/19 & $\Gamma / \mu \pm V \varepsilon / A F$ & ENQ \pm TERT. & $\Lambda \pm V / / Q V$ & تعادل يويا (يجهلو) \\
\hline$\cdot / \Delta V$ & $+1 \ldots *$ & $\Delta / \& A$ & $\Delta / \varphi \pm r N V^{e}$ & $N \Delta \pm A r / \& q$ & $|r / \Delta \pm r V / \Delta|$ & ثعادل ايستا \\
\hline
\end{tabular}

ــ "در سطح ه • P Pعنى Pار است. 
اصلاحشده به كار فتثه است.

\section{نتيجليَيرىنهايى}

با توجه به هدف كلى اين تحقيق كه بررسى ثأثير خستخى

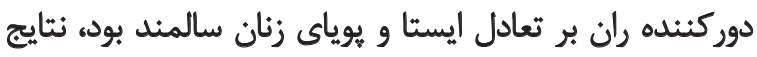

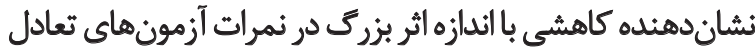

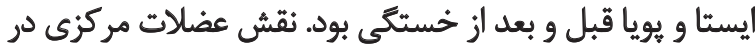

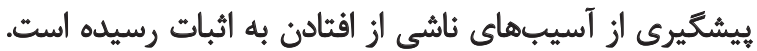

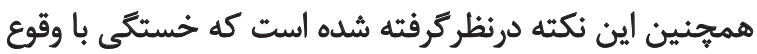

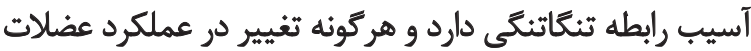

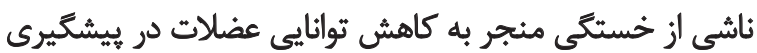

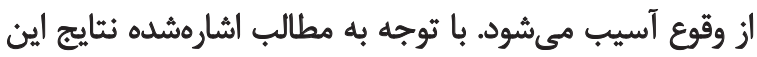

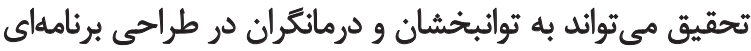

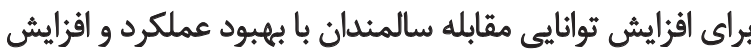
تعادل براى كاهش دفعات افتادكى كمك كنئد

يشئهانها

بيشنهاد مىشود در مطالعات آينده بهمنظور اطمينان از ايجاد

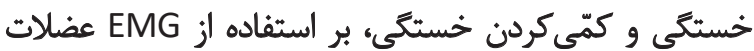

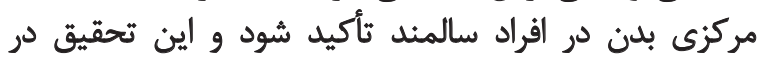
كروههاى سنى مختلف موردبررسى قرار كيرد.
(آزمون تعادل كردش ستاره در تمامي جهات) و همجينين آزمون

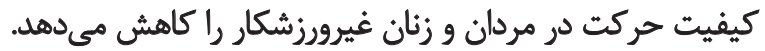

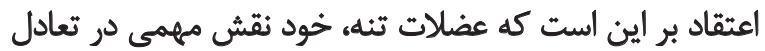

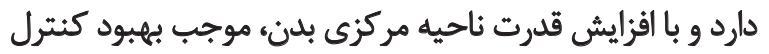

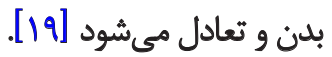

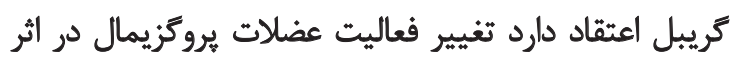

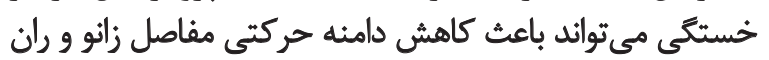

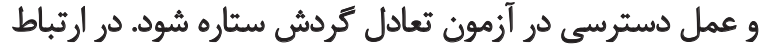

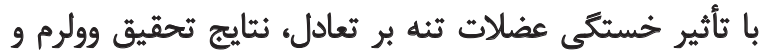

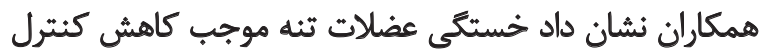

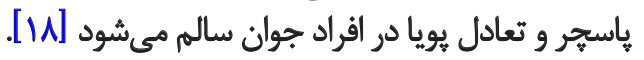
تحقيق شيخحسنى و همكاران با نتايج تحقيقات حاضر

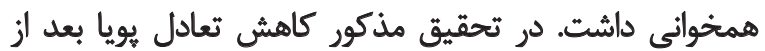

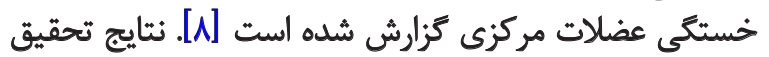

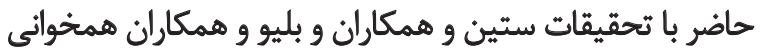

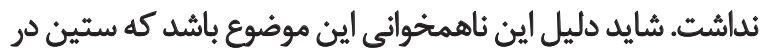

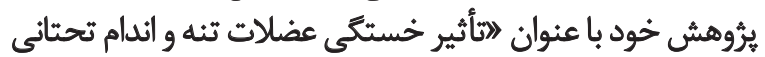

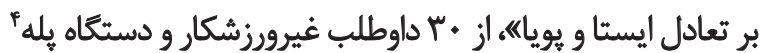

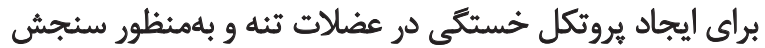

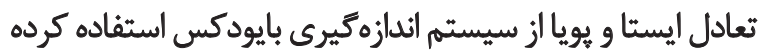

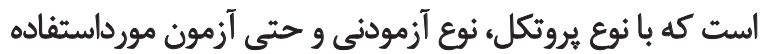

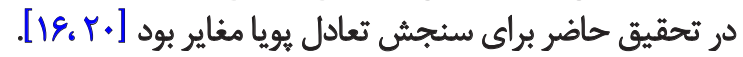
ستين معتقداست اين يروتكل خستكى موجب كاهش معنى دار

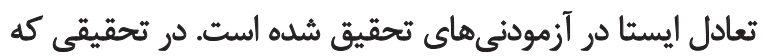

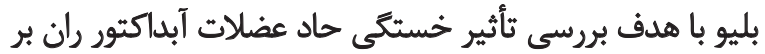

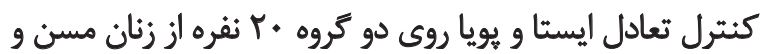

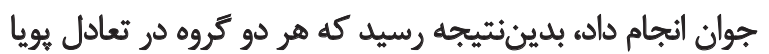

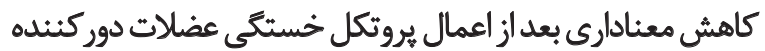

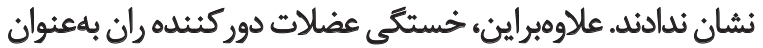

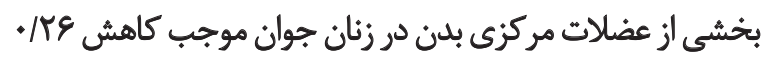

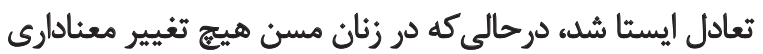

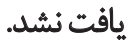

بليو و همكاران دليل اين عدمكاهش تعادل ايستا را افزايش

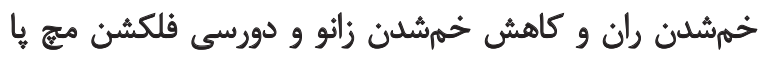

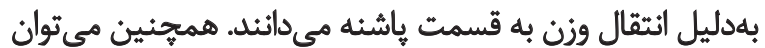

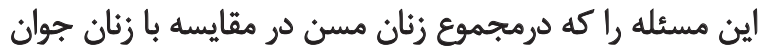

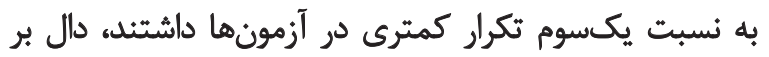

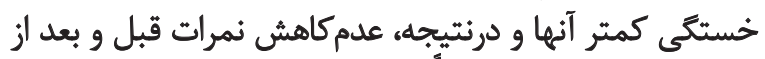

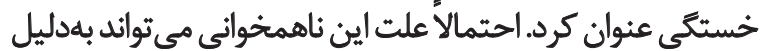

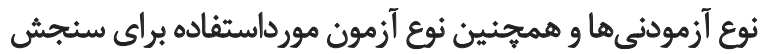

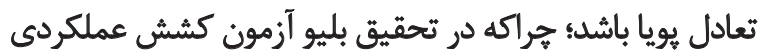




\section{References}

[1] Siqueira Rodrigues BG, Ali Cader S, Bento Torres NV, Oliveira EM, Martin Dantas EH. Pilate's method in personal autonomy, static balance and quality of life of elderly females. Journal of Bodywork Movement Therapies. 2010; 14(2):195-202.

[2] Clary S, Barnes C, Bamben D. Effects of ballates, step aerobics, and walking on medicine balance in women aged 50-75 years. Journal of Sports Science. 2006; 5(3):390-99.

[3] Foroughan M. [The reliable cognitive studies in the elderly (Persian)]. Tehran: Arjmand Press; 2009.

[4] Akbari Kamrani AA, Azadi F, Foroughan M, Siadat S, Kaldi A. [Charactristics of falls among institutionalized elderly people (Persian)]. Iranian Journal of Ageing. 2007; 1(3):101-05.

[5] Shumway-Cook A, Woollacott MH. Motor control: translating research into clinical practice. $3^{\text {rd }}$ ed. Philadelphia: Lippincott Williams \& Wilkins; 2006.

[6] Augustsson J, Thomee R, Karlsson J. Ability of a new hop test to determine functional deficits after anterior cruciate ligament reconstruction. Knee Surgery, Sports Traumatology, Arthroscopy. 2004; 12(5):350-56

[7] Augustsson J, Thomee R, Linden C, Folkesson M, Tranberg R, Karlsson J. Single leg hop testing following fatiguing exercise: reliability and biomechanical analysis. Scandinavian Journal of Medicine \& Science in Sports. 2006; 16(2):111-20.

[8] Sheikh-Hassani S, Rajabi R, Minoonejad H. The effect of core muscle fatigue on measurements of lower extremity functional performance in male athletes. Journal of Research in Rehabilitation Sciences. 2013; 9(4):668-82.

[9] Hass CJ, Gregor RJ, Waddell DE, Oliver A, Smith DW, Fleming $\mathrm{RP}$. The influence of Tai training on the center of pressure trajectory during gait initiation in older adult. Archives of Physical Medicine \& Rehabilitation. 2004; 85(10):1593-598.

[10] Bellew JW, Click Fenter P. Control of balance differs following knee or ankle fatigue in older women. Archives of Physical Medicine \& Rehabilitation. 2006; 87(11):1486-489.

[11] Waldhelm A, Li L. Endurance tests are the most reliable core stability related measurements. Journal of Sport and Health Science. 2012; 1(2):121-28.

[12] Earl JE. Gluteus medius activity during 3 variations of isometric single-leg stance. Journal of Sport Rehabilitation. 2005; 14:1-11. doi: 10.1186/1758-2555-2-17

[13] McMullen KL, Coby NL, Hertel J, Ingersoll CD, Hart JM. Lower extremity neuromuscular control immediately after fatiguing hip-abduction exercise. Journal of Athletic Training. 2011; 46(6):607-14

[14] Patrek MF, Kernozek TW, Willson ST, Wright GA. Hip-abductor fatigue and single-leg landing mechanics in women athletes. Journal of Athletic Training. 2011; 46(1):31-42.

[15] Gribble P, Hertel J, Phillip A. Considerations for the normalizing measures of the star excursion balance test. Measurement in Physical Education and Exercise Science. 2003; 7(2):89-100.

[16] Bellew J, Panwitz B, Peterson L, Brock M, Olson K. Effect of acute fatigue of the hip abductors on control of balance in young and older women. Archives of Physical Medicine \& Rehabilitation. 2009; 90(7):1170-175.
[17] Gribble PA, Hertel J. Effect of lower-extremity muscle fatigue on postural control. Archives of Physical Medicine and Rehabilitation. 2004; 85(4):589-92.

[18] Vuillerme N, Anziani B, Rougier P. Trunk extensor muscles fatigue affects undisturbed postural control in young healthy adults. Clinical Biomechanics. 2007; 22(5):489-94.

[19] Surenkok O, Kin-Isler A, Aytar A, Gültekin Z. Effect of trunkmuscle fatigue and lactic acid accumulation on balance in healthy subjects. Journal of Sport Rehabilitation. 2008; 17(4):380-86.

[20] Cetin N, Bayramoglu M, Surenkok AO, Yemisci OU. Effects of lower-extremity and trunk muscle fatigue on balance. Open Sports Medicine Journal. 2008; 2(1):16-22.

[21] Russell KA, Palmieri RM, Zinder SM. Differences in valgus knee angle during a single-leg drop jump. Journal of Athletic Training. 2006; 41(2):166-71.

[22] Akuthota V, Nadler SF. Core strengthening. Archives of Physical Medicine \& Rehabilitation. 2004; 85(3):86-92.

[23] Willson JD, Dougherty CP, Ireland ML, Davis IMC. Core stability and its relationship to lower extremity function and injury. Journal of American Academy of Orthopaedic Surgery. 2005; 13(5):316-25.

[24] Bove M, Faelliandet E, Tacchino A, Lofrano F, Cogo CE, Rug geri P. Postural control after a strenuous treadmill exercise. Journal of Neuroscience Letters. 2007; 418(3):276-81. 
